\title{
Bidirectional WDM-Radio over Fiber System with Sub Carrier Multiplexing Using a Reflective SOA and Cyclic AWGs
}

\author{
Fady I. El-Nahal \\ Electrical Engineering Department, Faculty of Engineering \\ Islamic University of Gaza \\ Gaza City, Gaza Strip
}

\begin{abstract}
A bidirectional SCM-WDM RoF network using a reflective semiconductor optical amplifier (RSOA) and cyclic arrayed waveguide gratings (AWGs) was proposed and demonstrated. The purposed RoF network utilizes Sub Carrier Multiplexed (SCM) signals for down-link and an on-off keying (OOK) signals re-modulated for up-link. In this paper, A $50 \mathrm{~km}$ range colorless WDM-ROF was demonstrated for both $1 \mathrm{Gbit} / \mathrm{s}$ downstream and upstream signals. The BER performance of our scheme shows that our scheme is a practical solution to meet the data rate and cost-efficient of the optical links simultaneously in tomorrow's ROF access networks.
\end{abstract}

Keywords- Radio over Fiber (RoF); wavelength-division multiplexing (WDM); Sub-carrier modulation (SCM); arrayed waveguide grating $(A W G)$.

\section{INTRODUCTION}

Recently, data capacity of wireless communication has been radically expanded from voices and simple messages to multimedia in order to satisfy various demands of system users with evolutionary future services. RoF systems could be the answer to many urgent needs of the telecommunication networks, as they could provide the necessary bandwidth for the transmission of broadband data to end-users, other benefits are low attenuation loss, and immunity to radio frequency interference [1-4]. The combination of sub-carrier multiplexing (SCM) and wavelength division multiplexing (WDM) is likely to play great role in these systems [5-8]. Wavelength division multiplexing (WDM) technique is used to simplify the network architecture by allowing different BSs to be fed using a single fiber. Each BS would be assigned its own wavelength and signals sent to different users serviced by a particular BS would be transmitted on that wavelength by means of SCM [9]. In a RoF system, Most of the signal processing processes (including coding, Multiplexing, and RF generation and modulation) are carried out By Central Office (CO), which makes the Base Station (BS) cost-effective. Therefore, RoF will become a key technology in the next generation mobile communication system [10-16].

For the uplink, the BSs must include an optical source, which is modulated by the mm-wave uplink radio signals. This approach results a high cost BS. Here we use a low cost uplink configuration, which eliminates the need for an expensive WDM source at the BS. This is accomplished by using a reflective semiconductor optical amplifier (RSOA) in the BS which replaces the high cost WDM source [17,18]. Many bidirectional RoF systems have been studied recently where a reflective semiconductor optical amplifier (RSOA) plays an important role. The RSOA can be used as a modulator and amplifier [9]. This approach avoids the need of stabilized a laser at each BS, this uplink configuration may be applied to other wireless networks such as $3 \mathrm{G}$ mobile communication systems. This system has the advantage of a simplified BS design but it brings the problem of dispersion caused fading that occurs when high frequency signals travel along fiber. Chromatic dispersion severely limits the transmission distance. Optical single sideband (OSSB) modulation techniques are used to overcome fiber dispersion effects $[2,9,14]$.

In this paper, we propose a new self-restorable architecture for bidirectional WDM-PON. It utilizes one different wavelength assignments and $1 \times \mathrm{N}$ AWGs, as 16 channels each channel of AWGs is coupled to Base stations (BSs) by using a reflective semiconductor optical amplifier (RSOA). This is for achieving sixteen 1-Gb/s downstream and upstream signals.

\section{WDM-RoF ARCHITECTURE}

Figure 1 shows the proposed WDM-RoF architecture for transmitting subcarrier multiplexing (SCM) encoded channels over a bidirectional single mode optical fiber $(50-\mathrm{km})$. At the central office $(\mathrm{CO})$, a series of narrow bandwidth continuous wave $(\mathrm{CW})$ with various wavelengths are modulated via a LiNbO3 Mach-Zehnder modulator using $1 \mathrm{~Gb} / \mathrm{s}$ non-return to zero (NRZ) downstream data to generate downstream signals. Downlink data signal is mixed with local oscillator signal (10$\mathrm{GHz}$ ) and a carrier generator having a number of $\mathrm{RF}$ subcarriers. The generated SCM signals are multiplexed by Arrayed Waveguide Grating (AWG) and sent over the bidirectional single-mode fiber (SMF). A circulator is used in the central office $(\mathrm{CO})$ to separate the downstream and upstream traffic. The SCM signals are de-multiplexed by AWG in remote node $(\mathrm{RN})$ where various wavelength lights are sent to different Base stations. Simple AWGs that support both dedicated wavelengths and power-splitting bandwidth sharing are used at the $\mathrm{CO}$ and the remote node $(\mathrm{RN})$. 


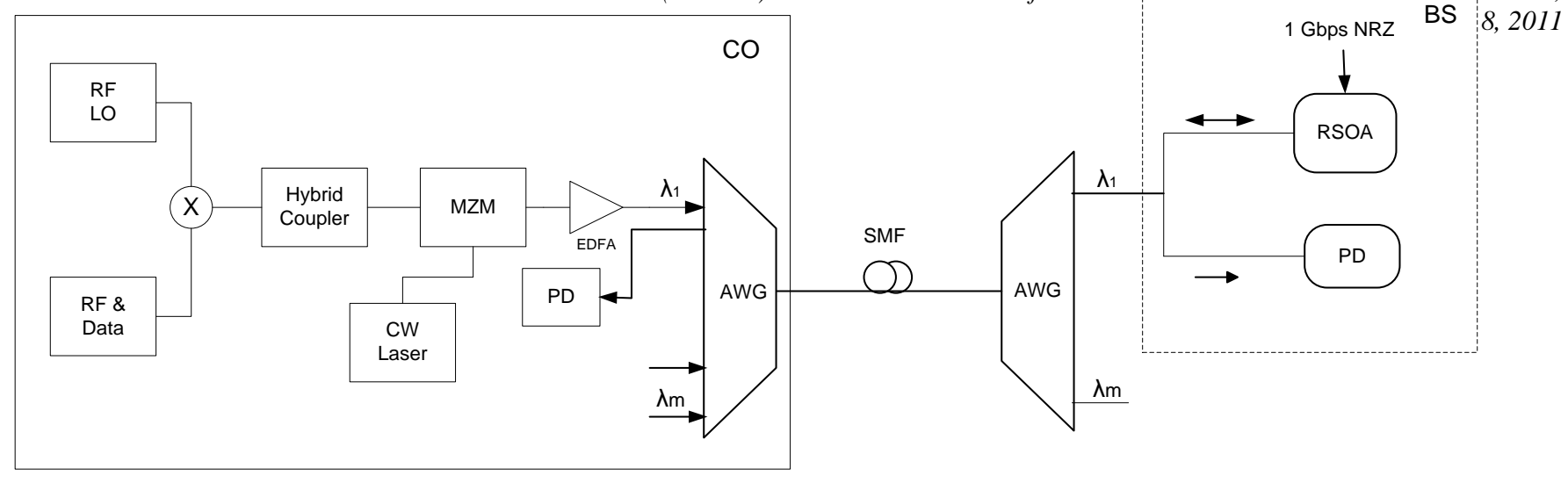

Figure 1: the proposed WDM-RoF architecture

At the BS, using optical splitter/coupler, portion of the SCM signal is fed to a SCM receiver. For up-link, the other portion of the downstream SCM signal from the splitter/coupler is re-modulated using $1 \mathrm{~Gb} / \mathrm{s}$ NRZ upstream data by RSOA in the BS. The re-modulated OOK signals are sent back over the fiber to the $\mathrm{CO}$ where they are demultiplexed by an AWG DEMUX. The reflected optical signal is detected by a PIN-photodiode. Uplink optical sidebands produce crosstalk when uplink data was detected at central station. Crosstalk can be reduced by using Bessel filter.

\section{RESULTS AND DISSCUSSIONS}

The WDM-RoF architecture was modeled using a commercially available package [19]. The proposed scheme uses SCM signal for downstream and OOK signal remodulated by the RSOA for upstream. The received eye diagrams of downstream and upstream signals were measured at Base station and central office respectively. The received eye diagrams of the downlink and uplink signals are shown in Figure 2 and Figure 3 respectively. The results show that the Eye closure penalty is smaller for the uplink than that of the downlink which is expected, as the signal travel twice the distance for the uplink. Chromatic dispersion induced by bidirectional fiber will not cause downlink microwave signal a power penalty problem. So, the Maximum eye amplitude for downlink stage after signal transmission took place over $50-\mathrm{km}$ of bidirectional fiber at base station.

BER simulations were carried out for both uplink and downlink with a Bit Rate of 1-Gb/s and no. of subcarriers $=70$. The BER variation with input optical power Pin curves for the downlink and uplink are shown in figure 4. It is clear that both uplink and downlink do provide good BER performances, however the BER results for the downlink are better than those of the uplink. For example, when $P_{\text {in }}=-5 \mathrm{dBm}$, the BER $=1.1 \times 10^{-11}$ for the downlink while it is $2.9 \times 10^{-10}$ for the uplink. When $P_{\text {in }}=+5 \mathrm{dBm}$, the BER $=8.8 \times 10^{-17}$ for the downlink while it is $1.1 \times 10^{-12}$

for the uplink. This can be attributed to the mixing noise between unsuppressed SCM signal in downstream and the digital signal of upstream which is generated in the remodulation process. This noise which influences the upstream signal could be reduced by using low pass filter after the photo detector in the CO. It is also noted from the figure that the BER for the uplink stays nearly constant for $P_{i n} \geq-1$ $\mathrm{dBm}$. This can be explained by the fact that the RSOA is operating in the gain saturation region. The variation of the gain of RSOA with the optical input power Pin is shown in figure 5. It is clear that the maximum gain appears at $P_{i n}=-5$ $\mathrm{dBm}$, then goes down to reach the lowest gain at $P_{i n}=-1 \mathrm{dBm}$ where it goes into saturation.

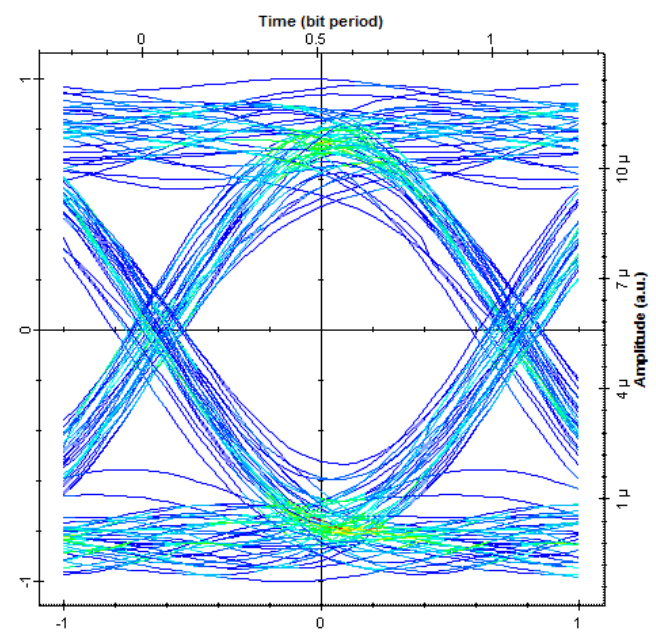

Figure 2: Eye diagram of downlink 


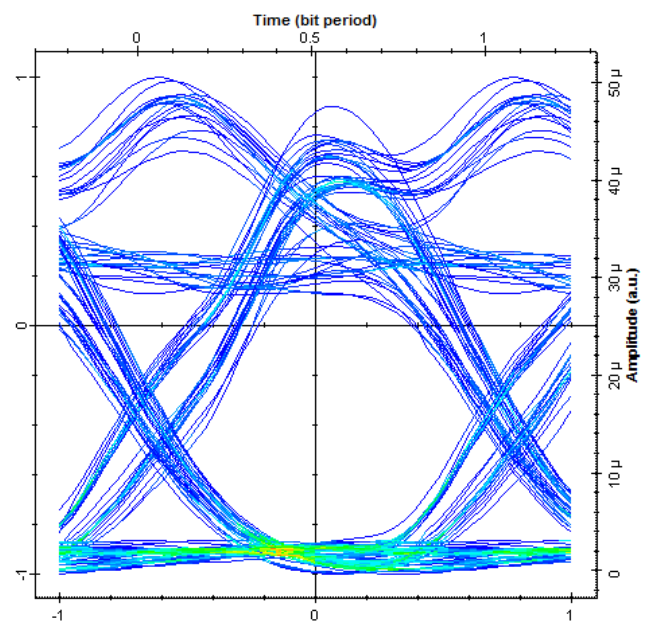

Figure 3: Eye diagram of uplink

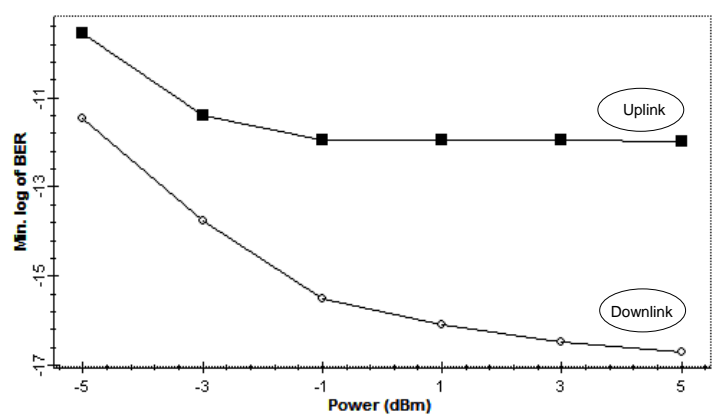

Figure 4: BER versus input power

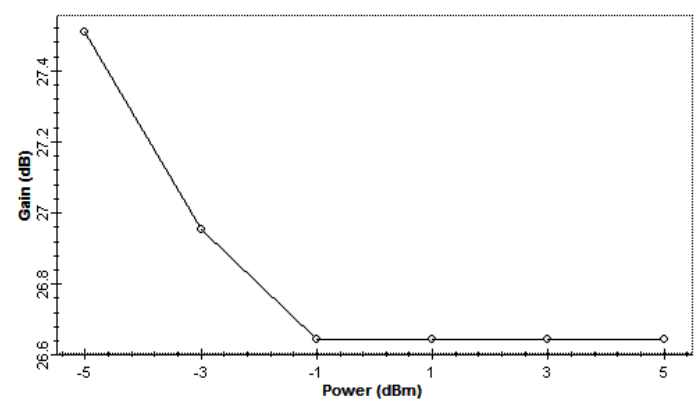

Figure 5: The variation of RSOA gain with input power.

The variation of BER with no. of subcarriers is plotted in Fig. 6. It is clear from the results that increasing the number of subcarrier channels degrade the system performance. For example, when RF channels increase from 10 to 90 , corresponding BER decreases from $4.6 \times 10^{-21}$ to $5.3 \times 10^{-14}$ for the downlink, while it decreases from $1.4 \times 10^{-15}$ to $7.3 \times 10^{-13}$ for the uplink. This can be explained by the fact that, as the number of sub-carrier channels increases, their frequency spacing decreases which results crosstalk or noise.

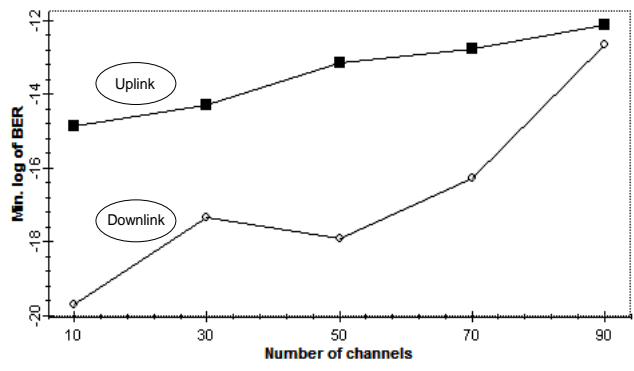

Figure 6: BER versus no. of channels

\section{CONCLUSION}

The WDM-RoF model has been proposed as solution for increased bandwidth demand. The combination of WDM and SCM has been performed to provide high data rates and bandwidth in wireless communication. In this paper we have analyzed the performance of WDM/SCM Radio over Fiber System. We presented a demonstration of $1 \mathrm{~Gb} / \mathrm{s}$ signal for up/downstream in $50-\mathrm{km}$ bidirectional link. The upstream traffic is obtained by remodulating the downstream traffic at the BS. The results obtained here show that increasing total number of sub-carriers channels has a significant impact on performance of WDM-SCM ROF system. The results show that $\mathrm{WDM} / \mathrm{SCM}$ system can play great role in future $\mathrm{RoF}$ systems.

\section{REFERENCES}

[1] H. Nasoha and S. M. Idrus" Modeling and Performance Analysis of WCDMA Radio over Fiber System Applied Electromagnetic", APACE 2007. Asia-Pacific Conference, December 2007 Melaka.

[2] Hyun-Seung Kim et al. , "Bidirectional WDM-RoF Transmission for Wired and Wireless Signals", Proc. of SPIE-OSA-IEEE Asia Communications and Photonics, SPIE Vol. 7632, 76322F, (2009).

[3] A Nirmalathas, P A. Gamage, C Lim, D Novak, and R Waterhouse, "Digitized Radio-Over-Fiber Technologies for Converged Optical Wireless Access Network", Journal of Lightwave Technology, Vol. 28, No. 16, pp. 2366-2375, (2010).

[4] Y.-M. Lin and P.-L. Tien, "Next-Generation OFDMA-Based Passive Optical Network Architecture Supporting Radio-Over-Fiber", IEEE Journal on Selected Areas in Communications - JSAC, vol. 28, no. 6, pp. 791-799, (2010).

[5] A. Kaszubowska, L.P. Barry, P. Anandarajah and Ling $\mathrm{Hu}$ "Characterization of Wavelength Interleaving in Radio-over-Fiber Systems Employing WDM/SCM", Optics Communications, 260 (1). pp. 144-149. ISSN 0030-4018, 2006.

[6] O.K. Tonguz et al., J. Lightwave Technol. 14 (1996) 1400.

[7] A. Stohr et al., Electron. Lett. 35 (1999) 1653.

[8] Fulvio Grassi, José Mora, Beatriz Ortega, José Capmany, "Experimental Evaluation of the Transmission in a Low Cost SCM/WDM Radio over Fibre System Employing Optical Broadband Sources and Interferometric Structures", in Proc. ICTON-09, pp. 1-4, São Miguel Island.

[9] M.C.R. Medeiros, R. Avó, P. Laurêncio, N.S. Correia, A. Barradas, H.J.A. da Silva, I. Darwazeh, J.E. Mitchell and P.M.N. Monteiro. "Radio 
[10] over Fiber Access Network Architecture Employing Reflective Semiconductor Optical Amplifiers", in Proc. ICTON-MW'07, pp. 1-5, Sousse, Tunisia.

[11] L. RAO, X. SUN, W. LI, D. HUANG "OFDM-ROF System and Performance Analysis of Signal Transmission" Optoelectronics, 2006 Optics Valley of China International Symposium on, Nov., 2006.

[12] R. Hui, Benyuan Z, Renxiang H., C. T. Allen, Kenneth R.D, and Douglas R. "Subcarrier Multiplexing for High-Speed Optical Transmission", Journal of Lightwave Technology, VOL. 20, NO. 3, MARCH, 2002.

[13] C. Loyez, C. Lethien, R. Kassi, J.P. Vilcot, D. Decoster, N. Rolland and P.A. Rolland "Subcarrier radio signal transmission over multimode fibre for $60 \mathrm{GHz}$ WLAN using a phase noise cancellation technique" ELECTRONICS LETTERS 20th Vol. 41 No. 2 Jan., 2005

[14] E. J. Tyler, P. Kourtessis, M. Webster, E. Rochart, T. Quinlan, S. E. M. Dudley, S. D. Walker, R. V. Penty, and I. H. White, "Toward Terabit-perSecond Capacities Over Multimode Fiber Links Using SCM/WDM Techniques “, Journal of Lightwave Technology, vol. 21, no. 12, Dec., 2003.

[15] A. Kaszubowska, P. Anandarajah, and L. P. Barry "Multifunctional Operation of a Fiber Bragg Grating in a WDM/SCM Radio Over Fiber Distribution System" IEEE Photonics Technology Letters, vol. 16, no. 2, Feb., 2004.

[16] S. M. Idrus,. "Photoparametric Amplifier in microwave Subcarrier Multiplexed Systems (PPA -in-SCM)", 22 Aug.,t 2007.

[17] T. Kim, K. Jeung-Mo, and H. Sang-Kook "performance analysis of bidirectional hybrid WDM/SCM PON link based on reflective semiconductor optical amplifier" Microwave and optical technology letters , ISSN 0895-2477, Vol. 48, April, 2006.

[18] Zhansheng Liu et al., "Experimental Validation of a Reflective Semiconductor Optical Amplifier Model Used as a Modulator in Radio Over Fiber Systems", IEEE Photon. Technol. Lett., vol. 23, no. 9, pp. 576-578, (2011).

[19] Yong-Yuk Won et al. , "Full Colorless WDM-Radio Over Fiber Access Network Supporting Simultaneous Transmission of Millimeter-Wave Band and Baseband Gigabit Signals by Sideband Routing", Journal of Lightwave Technology, vol. 28, no. 16, pp. 2213-2218, (2010).

[20] Optisystem from optiwave.

\section{AUTHORS PROFILE}

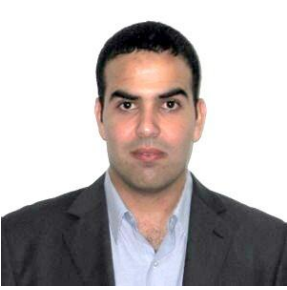

Dr. Fady I. El-Nahal received his B.Sc. degree in electrical and electronic engineering in 1996 from Alfateh University and his M.Phil. and Ph.D. degrees from the University of Cambridge in 2000 and 2004 respectively. $\mathrm{He}$ is currently with the Department of Electrical Engineering, The Islamic University of Gaza. His research activities include optoelectronics, optical communications and wavelength routing in optical networks. Fady is a Fellow of the Cambridge Overseas Trust and the chairman of the Oxford and Cambridge Society of Palestine. 